LBNL-47918

\title{
A PHYSICALLY-BASED ABRASIVE WEAR MODEL FOR COMPOSITE MATERIALS
}

Gun Y. Lee ${ }^{1,2}$, C. K. H. Dharan ${ }^{3}$, and R. O. Ritchie ${ }^{1,2}$

${ }^{1}$ Materials Sciences Division, Lawrence Berkeley National Laboratory

${ }^{2}$ Department of Materials Science and Engineering, University of California, Berkeley, CA 94720

${ }^{3}$ Department of Mechanical Engineering University of California, Berkeley, CA 94720

May 2001

submitted to Wear

Work supported by the Director, Office of Science, Office of Basic Energy Sciences, Materials Sciences Division of the U.S. Department of Energy under Contract No. DEAC03-76SF00098. 


\title{
A Physically-Based Abrasive Wear Model for Composite Materials
}

\author{
Gun Y. Lee ${ }^{1,3}$, C. K. H. Dharan ${ }^{1}$, and R. O. Ritchie ${ }^{2,3}$ \\ ${ }^{I}$ Department of Mechanical Engineering, University of California, Berkeley, CA $94720-1750$ \\ ${ }^{2}$ Department of Materials Science \& Engineering, University of California, Berkeley, CA 94720-1760 \\ ${ }^{3}$ Material Sciences Division, Lawrence Berkeley National Laboratory, Berkeley, CA 94720
}

SUMMARY: A simple physically-based model for the abrasive wear of composite materials is presented based on the mechanics and mechanisms associated with sliding wear in soft (ductile) matrix composites containing hard (brittle) reinforcement particles. The model is based on the assumption that any portion of the reinforcement that is removed as wear debris cannot contribute to the wear resistance of the matrix material. The size of this non-contributing portion of the reinforcement is estimated by modeling the three primary wear mechanisms, specifically plowing, interfacial cracking and particle removal. Critical variables describing the role of the reinforcement, such as its relative size and the nature of the matrix/reinforcement interface, are characterized by a single contribution coefficient, $C$. Predictions are compared with the results of experimental two-body (pin-on drum) abrasive wear tests performed on a model aluminum particulate-reinforced epoxy matrix composite material.

KEYWORDS : Composites, abrasive wear, interfacial toughness, size effect

\section{INTRODUCTION}

As advanced engineering materials, composites are used in many applications where high wear resistance is required; these include electrical contact brushes, cylinder liners, artificial joints, and helicopter blades. Indeed, compared to the monolithic materials, wear resistance can generally be enhanced by introducing a secondary phase(s) into the matrix material [1-6]. In this fashion, the wear properties can be varied substantially through changes in the microstructure, in terms of the morphology, volume fraction and mechanical properties of the reinforcing phase, and the nature of the interface between the matrix and reinforcement.

In order to obtain the optimum wear properties without compromising the beneficial properties in the matrix material, an accurate prediction of the wear of composites is essential. Unfortunately, for abrasive wear, existing models for composites are highly simplified and do not readily predict the role of the composite microstructure. In general, they are based on two simplified equations, the first of which, the inverse rule of mixtures, was introduced for twophase composites by Khruschov [7]:

$$
\frac{1}{W_{C}}=\frac{V_{M 1}}{W_{M 1}}+\frac{V_{M 2}}{W_{M 2}}
$$


where $W$ and $V$ are, respectively, the wear rates and volume fractions of the matrix (designated by subscript M1) and reinforcement (designated by M2). Note that the wear resistance, $R$, in Khruschov's original formulation is given by the reciprocal of the wear rate, $R=1 / W$.

Eq. (1) is based on the assumption that the components of the composite wear at an equal rate. Consequently, Khruschov's equation predicts that the abrasive wear resistance is linearly additive and that the wear resistance of the composite is simply the sum of the products of wear resistance and volume fraction for each component. Eq. (1) for a two-phase composite is plotted in Fig. 1. Since the wear rate of the harder reinforcement is typically much smaller than that of matrix, this relationship predicts that the abrasive wear behavior of a composite will be governed primarily by the reinforcement.

The second wear equation for multiphase materials, introduced by Zum-Gahr to explain experimental data, involves the linear rule of mixtures, where the wear behavior of a composite is not dominated by a single phase [6]. Instead, the contribution from each component is linearly proportional to its volume fraction in the composite:

$$
W_{C}=V_{M 1} \cdot W_{M 1}+V_{M 2} \cdot W_{M 2}
$$

as also plotted in Fig. 1. In this model, the abrasive wear rate of the composite decreases linearly with increasing volume fraction of reinforcement. This expression was derived by Axen and Jacobson using an equal pressure assumption that all components of a composite carry the same specific load [8]. A cyclic wear model for oriented fiber composites that predicted narrower bounds for abrasive wear behavior was proposed by Yen and Dharan [9]. In their paper, fiber instability due to preferential wear of the softer matrix resulted in cyclic generation of wear debris during the wear process. However, interface toughness and other physically-based factors were not considered in their model.

While Eqs. (1) and (2) are presumed to provide upper and lower limits for abrasive wear rates in a composite, this is not confirmed by some experimental results (Fig. 1) due to the simplified, non-physically based nature of the two models. Both rely on the notion that all components in the composite wear in the same way as they would in a bulk material; consequently the contribution of each component can depend only on its volume fraction and wear rate. The effects of other important factors, such as the interfacial properties between the distinctive phases and the relative sizes of these phases are not considered, even though it is clear that they have a significant influence on abrasion in composites [1,5,15-18]. Specifically, the wear rates of composites can exceed the bounds given by Eqs. (1) and (2) in that they are higher than that of the pure matrix material [1,10-14]; this implies that the presence of reinforcement enhances the damage instead of reducing it - the negative reinforcement effect. The inability to predict such effects represents a major limitation of existing abrasive wear models for composites.

In the present study, a new physically-based abrasive wear model for composite materials with hard reinforcements is developed based on three primary wear mechanisms: plowing, interfacial cracking and particle removal. The effects of critical factors, such as the interfacial properties and the relative size of the reinforcement, are specifically considered. The model provides estimates of the wear rate of the composite and predicts the occurrence of the negative reinforcement effect under certain conditions. Such predictions are verified by experimental abrasive wear studies conducted on a model aluminum particulate-reinforced epoxy matrix composite. 


\section{DUCTILE- AND BRITTLE-MATRIX COMPOSITES}

The sliding of abrasives on a solid surface results in volume removal. The mechanism of wear depends on the mechanical properties of the solid [19,20]. In a ductile solid, the primary wear mechanism is related to plastic deformation and the hardness of the material is a key parameter in governing the amount of material removal. However, the dominant mechanism in a brittle solid depends on fracture at or near the surface and the governing property is the toughness of the material.

To improve wear resistance, additional phase(s) can be introduced to either a ductile or a brittle material. However, the required mechanical properties of the reinforcement and the role of the reinforcement will be different in ductile vs. brittle matricies. For a ductile matrix, a hard secondary phase is needed to reduce wear, such that the presence of the hard reinforcement increases the effective hardness of the matrix, thereby reducing the penetration of the abrasive medium. Consequently, increasing the effective hardness acts to reduce the amount of material removed. Here, we term such a multiphase system composed of a ductile matrix and a hard reinforcement as a hard-reinforcement or hardened composite. On the other hand, a tough reinforcement phase is needed for a brittle matrix to increase wear resistance. The presence of a tough secondary phase reduces the tendency for fracture at or near the surface, and therefore tends to decrease the wear rate. In certain ceramic matrix composites, i.e., brittle matrix materials, the addition of a relatively ductile second phase can result in synergistically favorable wear behavior in which the composite wear rate can be less than the wear rates of the individual constituents. This is denoted by region B in Fig. 1 and has been observed in ceramic composites $[2,3]$. A multiphase system composed of a brittle matrix and a tough reinforcement may be termed a ductile-reinforcement or toughened composite.

The present study is focused on hard-reinforcement particulate composites, which have been the object of most modeling studies of the wear of composites in the past [e.g., 6,7]. We will consider reinforcement volume fractions in the range 0 to 0.5 , since at higher volume fractions one can expect significant particle-particle interactions. From a practical standpoint, it is difficult to manufacture particulate composites at volume fractions greater than 0.5 due to void formation and agglomeration.

\section{ABRASIVE WEAR MODEL}

A model has been developed with simplified geometry in two dimensions, namely a triangular abrasive medium particle acting on a composite containing idealized rectangular reinforcements. The model is based on the "equal wear rate assumption"; this postulates that the different components of a composite wear at steady state at an equal rate through the redistribution of the specific loads [7,21]. A general schematic drawing of a two-phase composite with a ductile matrix and a hard reinforcement in abrasion is shown in Fig. 2. The characteristic size of the reinforcement is represented by the parameter $D_{R}$.

If the fracture toughness of the matrix/reinforcement interface exceeds the minimum toughness of either constituent (a "strong" interface), then plowing will be the predominant wear mechanism; consequently, the resulting wear debris will be small in relation to the reinforcement size. With such strong interfacial bonding, the entire reinforcement particle will contribute to improving wear resistance. Both rules of mixtures are commonly based on this assumption. In practice, however, the interfacial bonding between the constituent materials may not be this 
strong, due to chemical incompatibility, the mismatch in thermal expansion, and elastic properties, e.g., stiffness, at the interface [22], and the presence of impurities and/or voids that arise during fabrication. This causes the motion of the abrasive medium to induce interfacial failure and debonding around the particles, which results in a portion of the reinforcement being removed as wear debris in the form of a large mass.

Since the portion of the reinforcement that is removed due to interfacial failure cannot further contribute to improving the wear properties of the matrix; its contribution to the wear resistance is inversely proportional to its relative size. This size can be estimated by modeling the three primary abrasive wear mechanisms, namely plowing, interfacial cracking and particle removal. Based on this information, a new relationship for the abrasive wear rate of a composite is developed.

\section{A. Plowing mechanism}

The depth of penetration, $x$, of the abrasive medium depends on its geometry, the applied normal load, and the mechanical properties of a composite (relative to the abrasive medium). While the abrasive medium is moving, contact with the substrate occurs only over its half-front surface. Under an indentation load $L$, the depth of penetration of the abrasive particle can be written as:

$$
\begin{gathered}
L=\frac{1}{2}\left(2 w \cdot b \cdot H_{C}\right) \text { and } \frac{x}{w}=\tan \theta \\
x=\frac{L}{b \cdot H_{C}} \tan \theta
\end{gathered}
$$

where $b$ is the thickness of the substrate and abrasive medium, and $H_{C}$ is the hardness of the composite. The magnitude of plowing load $F_{p}$ required to plastically deform and remove material is proportional to the depth of penetration of the abrasive medium. The abrasive medium will plow the matrix and the reinforcement alternatively, and experience different plowing loads for the different phases (Fig. 3). The expression for the plowing load on each phase can be expressed by employing the indentation load approximation:

$$
F_{p}=x \cdot b \cdot H_{i}
$$

where $H_{i}$ is the hardness of either the matrix or reinforcement material.

If the spacing, $d$, between individual abrasive particles is small compared to the extent of their respective stress fields, then an interaction between neighboring stress fields will occur (Fig 3 ). Consequently, the net tensile stress, $\sigma^{+}$, behind each abrasive particle will depend on the average distance between these particles and the magnitude of the plowing loads:

$$
\left(\sigma^{+}\right)_{n}=f\left\{\left(F_{P}\right)_{n}, d,\left(F_{P}\right)_{n+1}\right\}
$$

\section{B. Interfacial cracking}

If the fracture toughness of the matrix/reinforcement interface is significantly less than that of the matrix (a "weak" interface), plowing by the abrasive medium can lead to the propagation of 
an interfacial crack (Fig. 4). The maximum tensile load is applied at the matrix/reinforcement interface when the abrasive medium plows the reinforcement, i.e.,

$$
\left(F_{p}\right)_{n}=\left(F_{p}\right)_{R}
$$

At this point, the size, $l$, of the interfacial crack will be a function of the net tensile stress and the relative toughness of the interface and the matrix:

$$
l=g\left\{\frac{1}{G_{i f}},\left(\sigma^{+}\right)_{n}\right\}
$$

where $G_{i f}$ is the interface fracture toughness. By combining Eqs. (5) and (7), the length of the interfacial crack can be expressed as:

$$
l=g\left\{\frac{1}{G_{i f}},\left(F_{P}\right)_{R}, d,\left(F_{P}\right)_{n+1}\right\}
$$

\section{Particle removal}

In a given tribo-system, the size of the interfacial crack $l$ under the maximum tensile load is assumed to be constant. The continuous plowing of the abrasive particles reduces the level of the surface until the tip of the interfacial crack finally reaches the bottom of the reinforcement (Fig. $5(\mathrm{a}))$.

Continued plowing of the abrasive medium causes further propagation of the interfacial crack, its trajectory depending on the relative toughness of the interface. When the ratio of the fracture toughness of the interface and the matrix is less than approximately 0.25 for this geometry (this ratio does vary with the orientation of the crack), the interfacial crack will deflect along the interface [23], i.e., when:

$$
\frac{G_{i f}}{G_{m}}<\frac{1}{4}
$$

where $G_{i f}$ and $G_{m}$ are, respectively, the fracture toughness of the interface and matrix material.

Further motion of the abrasive medium can cause the complete removal of the remaining portion of the reinforcement leaving a void of the same size on the surface. As a portion of reinforcement is now removed as a large mass (due to the interfacial failure), it cannot contribute to the wear resistance. The fraction of this "non-contributing portion" is:

$$
\frac{(x+l)}{D_{R}}
$$

where $x$ is the depth of penetration of the abrasive medium, $l$ is the size of the interfacial crack, and $D_{R}$ is the size of the reinforcement (Fig. 5(b)). 
In terms of these three primary wear mechanisms, the wear behavior of a two-phase composite is akin to that of a three-phase composite composed of a matrix, reinforcement and pores. The volume fraction of the porous section is equal to the volume fraction of the noncontribution portion of the reinforcement, which is the product of volume fraction of reinforcement $V_{R}$ and the fraction of the non-contributing portion (Eq. (11)).

$$
V_{\text {pore }}=V_{R} \cdot\left(\frac{(x+l)}{D_{R}}\right) \quad V_{R} \leq 0.5
$$

Thus, the net volume fraction of the reinforcement, which contributes to the wear resistance can be written as:

$$
V_{n e t}=V_{R} \cdot\left(1-\frac{(x+l)}{D_{R}}\right) \quad V_{R} \leq 0.5
$$

The wear rate of the three-phase composite can again be obtained based on the "equal wear rate assumption":

$$
\frac{1}{W_{C}}=\frac{V_{m}}{W_{m}}+C \cdot \frac{V_{R}}{W_{R}}+(1-C) \cdot \frac{V_{R}}{W_{\text {pore }}} \quad V_{R} \leq 0.5
$$

where $W_{c}, W_{m}, W_{R}$ and $W_{\text {pore }}$ are the wear rates of composite, matrix, reinforcement and pores, $V_{m}$ and $V_{R}$ are the volume fractions of the matrix and the reinforcement, and $C$ is a new parameter, which we term the contribution coefficient of reinforcement. This parameter describes the relative contribution of each of the primary wear mechanisms and is defined by the size of the reinforcement, the plowing depth and the interfacial crack size as:

$$
C=\left(1-\frac{(x+l)}{D_{R}}\right)
$$

Since the third term on the right side of Eq. (13) will vanish because the wear resistance of pores is equal to zero, $1 / W_{\text {pore }}=0$, we can obtain a final expression for the abrasive wear rate of a composite from this physically-based mechanistic model as:

$$
\frac{1}{W_{C}}=\frac{V_{m}}{W_{m}}+C \cdot \frac{V_{R}}{W_{R}} \quad V_{R} \leq 0.5
$$

The contribution coefficient parameter represents the effects of critical factors, including the interfacial toughness and the relative size of the reinforcement; its magnitude varies from zero to unity. 


\section{EXPERIMENTS}

\section{A. Materials}

To provide some degree of experimental verification of the proposed model, abrasive wear tests were conducted with a model composite system involving an epoxy matrix with spherical aluminum alloy particulate reinforcement. In order to minimize the effect of the mismatch in coefficient of thermal expansion, a room temperature curing epoxy was selected as the matrix material; this was DER 331 epoxy resin and DEH 24 hardener from Dow Chemical, Midland, MI USA. The epoxy was reinforced with 6061aluminum metal particles provided by Valimet Inc., Stockton, CA USA; the particles were nominally spherical with an average size of $\sim 100 \mu \mathrm{m}$.

In order to vary the contribution coefficient $\mathrm{C}$ in Eq. (15), tests were performed for composites with different matrix/reinforcement interfacial toughnesses and with different relative sizes of reinforcement. A "strong" interface was achieved by reinforcing the epoxy with uncontaminated particles, whereas a "weak" interface was achieved by prior coating of the particles with a thin layer of silicone. By conducting tests on different sizes of abrasive (35 326 $\mu \mathrm{m})$, the relative size of reinforcement was varied.

Tests were performed for the specimens with $0,20,40$ and 100 vol.\% of reinforcement. The composites were fabricated by the stir-casting method, specifically involving the mixing of metal particles in a liquid epoxy followed by casting in an open mold $(9.5 \mathrm{~mm}$ diameter and $20 \mathrm{~mm}$ length) in a vacuum.

\section{B. Abrasive wear test}

Two-body abrasive wear tests were conducted on a pin-on drum abrasive wear tester, designed for standard wear tests described ASTM standard D5963-97a. In this method, the test specimen moves over the surface of an abrasive, which is located on a revolving drum, the resulting wear of the material being expressed as the volume loss [24]. The test setup is schematically illustrated in Fig. 6.

An alumina $\left(\mathrm{Al}_{2} \mathrm{O}_{3}\right)$ abrasive was used which is substantially harder than the matrix and reinforcement. The pin specimen, which was $0.95 \mathrm{~mm}$ diameter and $20 \mathrm{~mm}$ long, was placed on the top of the drum, which was then rotated at a fixed angular speed of 25 RPM; this gives a tangential velocity at the contact surface of $200 \mathrm{~mm} / \mathrm{s}$. While the drum was rotating, the specimen moves at the speed of $4.2 \mathrm{~mm} / \mathrm{rev}$ along the axis of rotation. Thus, the specimen is continuously in contact with new abrasive surface. A static normal load, $L$, was applied directly above the specimen to press it against the center of the drum (Fig. 6); its magnitude was varied from 1 to $5 \mathrm{~N}$, corresponding to normal stress from 13.8 to $68.8 \mathrm{kPa}$. Throughout the test, the sliding distance was fixed at $39.2 \mathrm{~m}$ (80 revolutions). All tests were carried out in dry ambient air laboratory conditions.

\section{RESULTS AND DISCUSSION}

\section{A. Prediction with the new model}

Predicted wear rates from Eq. (15) for the two ideal composites with different contribution coefficients and volume fractions are shown in Fig. 7. The two composites, termed composite 1 
and 2, differ in their relative wear resistance; the abrasive wear resistance of the reinforcement in composite 2 is twice as large as that of the reinforcement in composite 1.

From Fig. 7, predictions of the wear rates of the composites at a fixed volume fraction can be obtained. These wear rates depend strongly on the contribution coefficient, $C$, of the reinforcement. When $C=1$, i.e., in the absence of particle removal such that the reinforcement wears the same way as if it were in bulk, the size of the non-contributing portion of the reinforcement, $(x+l)$ in Fig. 5 (b), becomes small compared to the total size of the reinforcement $D_{R}$. Under these conditions, the model predicts wear rates that are equal to the lower limit of the inverse law of mixtures model (Eq. (1)). As the contribution coefficient is reduced, wear rates are predicted to increase above this lower limit; indeed, as $C \rightarrow 0$, predicted wear rates of the composites become higher than that of the matrix, thus providing a justification for the negative reinforcement effect.

As the value of $C$ depends strongly on the penetration of the abrasive medium $x$ and the interfacial crack size $l$, the toughness of the matrix/reinforcement interface is a critical factor governing the magnitude of $l$. If the interfacial toughness is reduced, the size of the interfacial crack increases and thus the value of $C$ is decreased. Consequently, the model predicts that the wear rate of the composite will be increased by any reduction in the interfacial toughness. A corresponding increase in the depth of penetration will effectively reduce the relative size of the reinforcement. This lowers the value of $C$ and consequently increases the wear rate of the composite. In contrast, raising the interfacial toughness and decreasing the depth of penetration will enhance the contribution of the reinforcement and thus reduce the wear rate of the composite.

Another noticeable observation from these predictions is that even though composite 2 has a more wear-resistant reinforcement, the overall wear resistance of the composite depends also on factors such as the interfacial toughness and relative reinforcement size, i.e., on the value of $C$. Thus, the present model highlights the fact that choosing a reinforcement solely on its bulk wear resistance without considering these other factors will not guarantee the optimal wear characteristics of the composite itself. For example, at a reinforcement volume fraction, $V_{R}=0.5$, the critical value of $C$ below which the wear rate exceeds the upper bound is about $C=0.28$ in the composite with the more wear resistant reinforcement (Composite 2, Fig. 7 (b)), while $C$ can be a large as 0.5 for the composite containing the less wear resistant reinforcement for the wear rate to exceed the upper bound. That is, a composite with a more wear resistant reinforcement can tolerate a lower level of interface toughness than a composite containing a less wear resistant reinforcement. This result is consistent with experimental observations made by other investigators [1,10-14], and indicates that the proposed coefficient $C$ has a strong physical basis for the class of hard reinforcement composites considered here.

\section{B. Experimental results}

The results of the pin-on-drum two-body abrasive wear tests are shown in Figs. 8 and 9. The interfacial toughness and the relative size of the reinforcement were changed to effect a variation in the contribution coefficient $C$. Test results for the composites with different interfacial conditions, in the form of "strong" and "weak" interfaces, are displayed in Fig. 8.

For both composites, the experimental results are in reasonable agreement with the model predictions using contribution coefficients of $C=0.4$ for the "strong interface" composite and $C$ $=0$ for the "weak interface" material. Such predictions are consistent with observations that poor interfacial toughness induces large interfacial crack sizes and hence a low contribution of the reinforcement. 
Since the relative size of the reinforcement is small in particulate-reinforced composites, the effect of the interfacial toughness on the wear rate can be significant. For example, when the interface is weak, the reinforcement can be readily removed during abrasive wear conditions, such that a negative reinforcement effect is observed. Indeed, as shown in Fig. 8 (b), the wear rates in the composite were higher than those in the unreinforced matrix; moreover, with additional reinforcement, the wear rates were further increased.

The abrasive wear rates of composites with different relative sizes of the reinforcement (relative to the abrasive medium) are plotted in Fig. 9. It can be seen that for the case of a large reinforcement particle size relative to abrasive size, which corresponds to classical abrasive wear conditions, a value of $C=0.4$ shows good agreement with the experimental results (Fig. 9 (a)). The experimental data fall within the rules of mixtures bounds, exceeding the upper bound at a reinforcement volume fraction of about 0.5 . When the reinforcement particle is small relative to the abrasive size, however, the wear rates increase considerably for the same composite material (Fig. 9 (b)). This is due to plowing by the abrasive medium, leading to a higher wear rate asociated with debonding and particle removal mechanisms. This physically-based consideration is not in the rules of mixtures formulations proposed earlier. Once again, the test results are consistent with the model's predictions.

\section{CONCLUSIONS}

A simple framework for a physically based model for abrasive wear in ductile composites reinforced with a hard second phase is presented based on the salient mechanisms of sliding wear, namely plowing, interface cracking, and particle removal. The model relies on the straightforward notion that any portion of reinforcement that is removed as wear debris cannot contribute to the wear resistance of the material; the size of this non-contributing portion of the reinforcement is estimated from the mechanistic descriptions. Critical variables describing the role of the reinforcement are identified in terms of the relative size of the reinforcement, the depth of plowing and the toughness of the matrix/reinforcement interface. The model provides a reasonable description of the variation in abrasive wear rates with reinforcement volume fraction and provides a justification for the "negative reinforcement" effect.

\section{ACKNOWLEDGMENTS}

Support for one of the authors (ROR) was provided by the Director, Office of Science, Office of Basic Energy Sciences, Materials Sciences Division of the U.S. Department of Energy under Contract No. DE-AC03-76SF00098.

\section{REFERENCES}

1. K.J. Bhansali and R. Mehrabian, Abrasive wear of aluminum-matrix composites, Journal of Metals, 34 (1982) 30-34.

2. C.P. Dogan and J.A. Hawk, Role of zirconia toughening in the abrasive wear of intermetallic and ceramic composites, Wear, 212 (1997) 110-118.

3. D.Holz, R. Janssen, K. Friedrich and N. Claussen, Abrasive wear of ceramic-matrix composites, Journal of the European Ceramic Society, 5 (1989) 229-232.

4. M.M. Khruschov, Principles of abrasive wear, Wear, 28 (1974) 69-88. 
5. S. Skoliannos and T.Z. Kattamis, Tribological properties of SiC/sub p/-reinforced Al-4.5\% $\mathrm{Cu}-1.5 \% \mathrm{Mg}$ alloy composites, Materials Science \& Engineering A (Structural Materials: Properties, Microstructure and Processing), 163 (1993) 107-113.

6. K.H. Zum-Gahr, Abrasive wear of two-phase metallic materials with a coarse microstructure, in: K.C. Ludema (ed.), International Conference on Wear of Materials, American Society of Material Engineering, Vancouver, 1985, 793 p.

7. M.M. Khruschov and M.A. Babichev, Resistance to abrasive wear of structurally heterogeneous materials, Friction and Wear in Machinery, 12 (1958) 5-24.

8. N. Axen and S. Jacobson, A model for the abrasive wear resistance of multiphase materials, Wear, 174 (1994) 187-199.

9. B. Yen and C. K. H. Dharan, A model for the abrasive wear of fiber-reinforced polymer composites, Wear, 195 (1996) 123-127.

10. N. Axen and K.H. Zum-Gahr, Abrasive wear of TiC-steel composite clad layers on tool steel, Wear, 157 (1992) 189-201.

11. N. Axen, A. Alahelisten, and S. Jacobson, Abrasive wear of alumina fibre-reinforced aluminium, Wear, 173 (1994) 95-104.

12. N. Axen and S. Jacobson, Transitions in the abrasive wear resistance of fibre- and particlereinforced aluminium, Wear, 178 (1994) 1-7.

13. F.M. Hosking, F. Folgar Portillo, R. Wunderlin and R. Mehrabian, Composites of aluminium alloys: fabrication and wear behaviour, Journal of Materials Science, 17 (1982) 477-498.

14. S.V. Prasad and P.D. Calvert, Abrasive wear of particle-filled polymers, Journal of Materials Science, 15 (1980) 1746-1754.

15. S.-J. Liu, and K.-S. Lin, Effect of aging on abrasion rate in an Al-Zn-Mg-SiC composite, Wear, 121 (1988) 1-14.

16. S.V. Prasad and P.K. Rohatgi, Tribological properties of Al alloy particle composites, Journal of Metals, 39 (1987) 22-26.

17. W. Simm and S. Freti, Abrasive wear of multiphase materials, Wear, 129 (1989) 105-121.

18. A.C.M. Yang, J.E. Ayala, A. Bell and J.C. Scott, Effects of filler particles on abrasive wear of elastomer-based composites, Wear, 146 (1991) 349-366.

19. I.M. Hutchings, Tribology; Friction and wear of engineering materials, Arnold, London, $1992,273 \mathrm{p}$.

20. K.-H. Zum-Gahr, Microstructure and wear of materials. Tribology Series 10, Elsevier Science, New York, 1987, $560 \mathrm{p}$.

21. W.M. Garrison, Khruschov's rule and the abrasive wear resistance of multiphase solids, Wear, 82 (1982) 213-220.

22. S.R. White and R.G. Albers, Experimental investigation of aluminum/epoxy interfacial properties in shear and tension, Journal of Adhesion, 55 (1996) 303-316.

23. M.-Y. He and J.W. Hutchinson, Crack deflection at an interface between dissimilar elastic materials, International Journal of Solids Structures, 25 (1989) 1053-1067.

24. Annual Book of ASTM Standards, American Society for Metals, West Conshohocken, PA, $1999,544 \mathrm{p}$. 


\section{FIGURE CAPTIONS}

Fig. 1: Predicted abrasive wear rates of composites. Eqs. (1) and (2) represent the lower and upper limits of the existing methods. Experimental results (region A) of composites reinforced with hard particles often lie outside these bounds [1,9-13].

Fig. 2: General schematic drawing of a two-phase composite in abrasion.

Fig. 3: Abrasive particles plow matrix and reinforcement alternatively and stress fields are formed around them; compressive stresses are formed in front and tensile stresses behind the abrasive particle.

Fig. 4: Delamination of the matrix/reinforcement interface leading to interfacial crack propagation under the tensile loading generated by sliding motion of the abrasive particle.

Fig. 5: A portion of the reinforcement is removed due to interface failure.

Fig. 6: Pin-on drum abrasive wear test set-up. The drum support is $150 \mathrm{~mm}$ in diameter and rotates at $25 \mathrm{rpm}$. The longitudinal traverse is $4.2 \mathrm{~mm} / \mathrm{rev}$ of the drum. A counter records the number of revolutions. The composite specimen is loaded onto the drum with a specified weight.

Fig. 7: Prediction of abrasive wear rates, normalized to the wear rate of the reinforcement, for two ideal composites with different contribution coefficients and volume fractions. The two composites, termed composite 1 and 2, differ in their relative wear resistance; the abrasive wear resistance of the reinforcement in composite 2 is twice as large as that of the reinforcement in composite 1 . Plots are for various values of the contribution coefficient, $C$. The shaded region represents the predictions from the rule of mixtures models, specifically Eqs. (1) and (2).

Fig. 8: Abrasive wear rates of particulate reinforced model composites with different interfacial properties between matrix and reinforcement, showing predictions for a) a strong interface $\left(l<<D_{R}\right)$, and b) a very weak interface $\left(l \sim D_{R}\right)$. The curve through the experimental results for Al-reinforced epoxy matrix composites are shown as a solid line; predictions are dashed lines. The shaded region represents the predictions from the rule of mixtures models, specifically Eqs. (1) and (2).

Fig. 9: Abrasive wear rates with different relative size of the reinforcement relative to the abrasive medium, showing experimental results for Al-reinforced epoxy composites (solid lines with data points) and model predictions (dashed lines) for composites with a) large $\left(D_{R}>x\right)$ reinforcements, and b) small $\left(D_{R} \sim x\right)$ reinforcements. The shaded region represents the predictions from the rule of mixtures models, specifically Eqs. (1) and (2). 


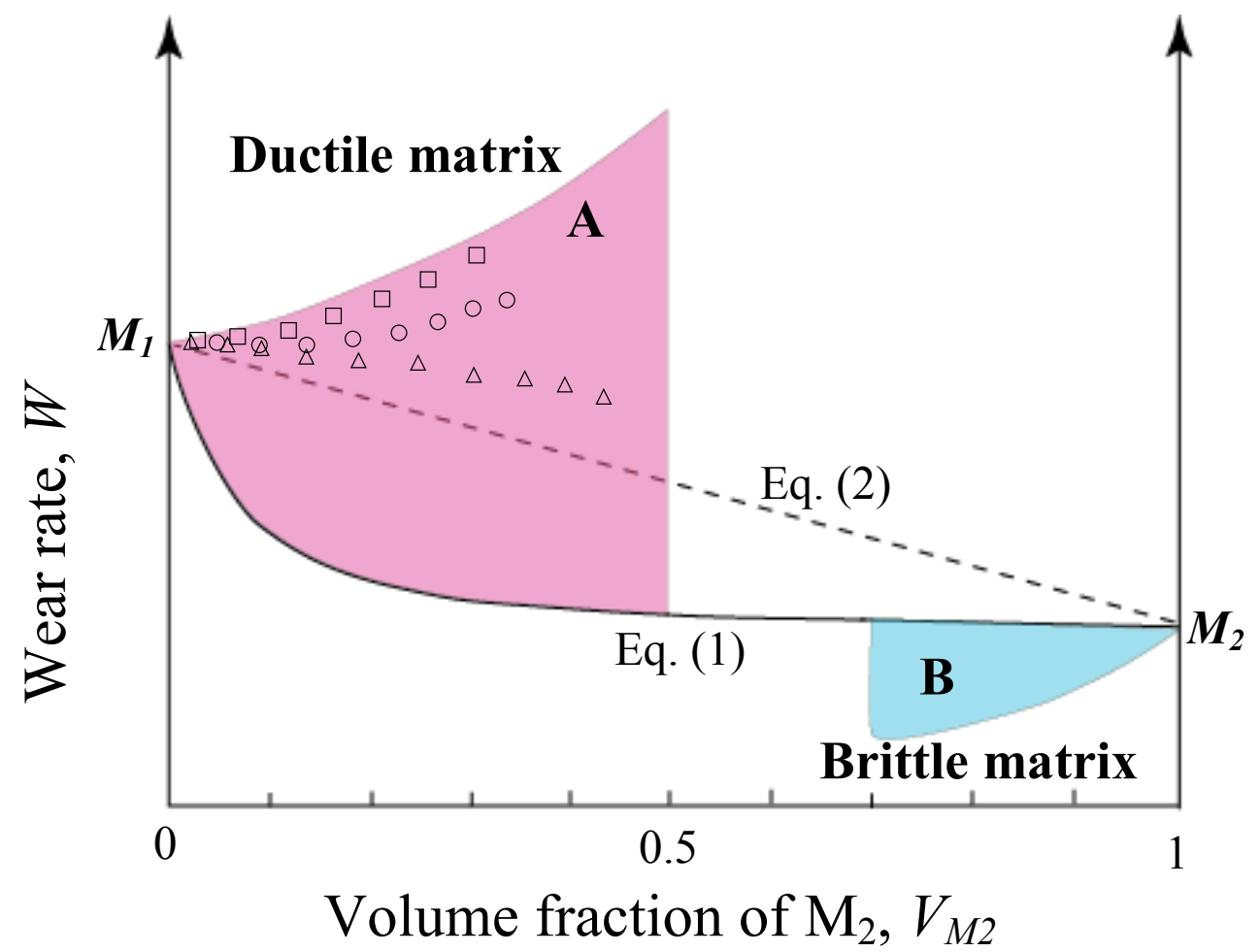

Fig. 1: Predicted abrasive wear rates of composites. Eqs. (1) and (2) represent the lower and upper limits of the existing methods. Experimental results (region A) of composites reinforced with hard particles often lie outside these bounds [1,9-13].

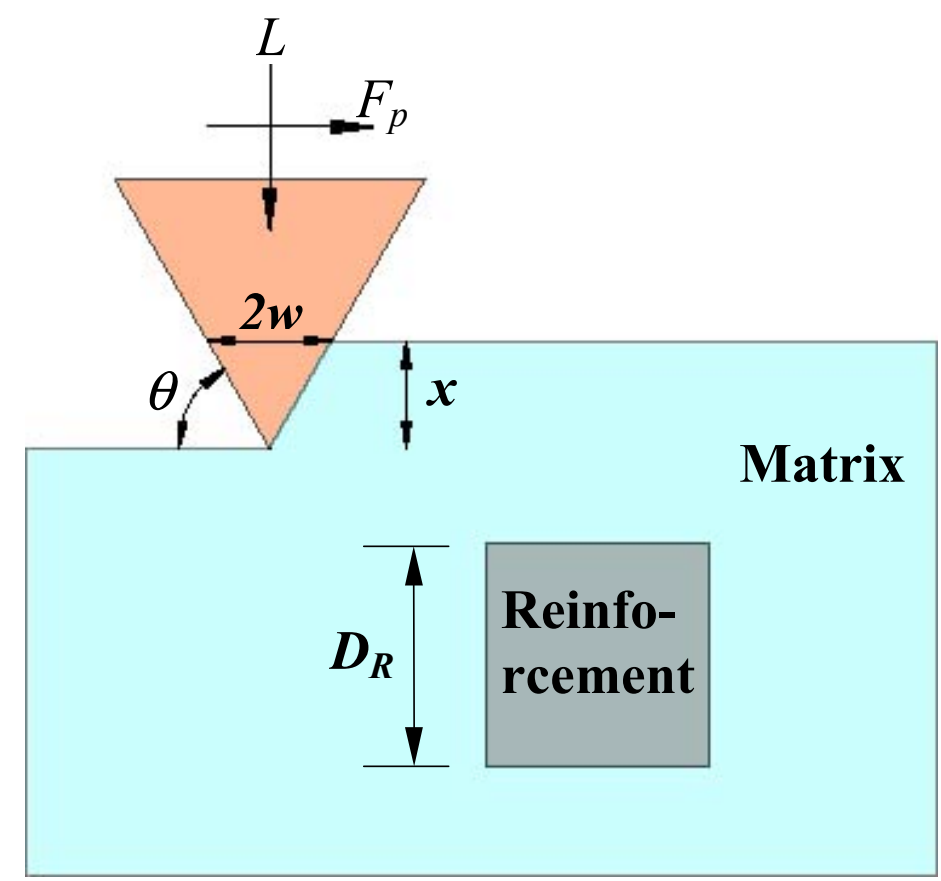

Fig. 2: General schematic drawing of a two-phase composite in abrasion. 


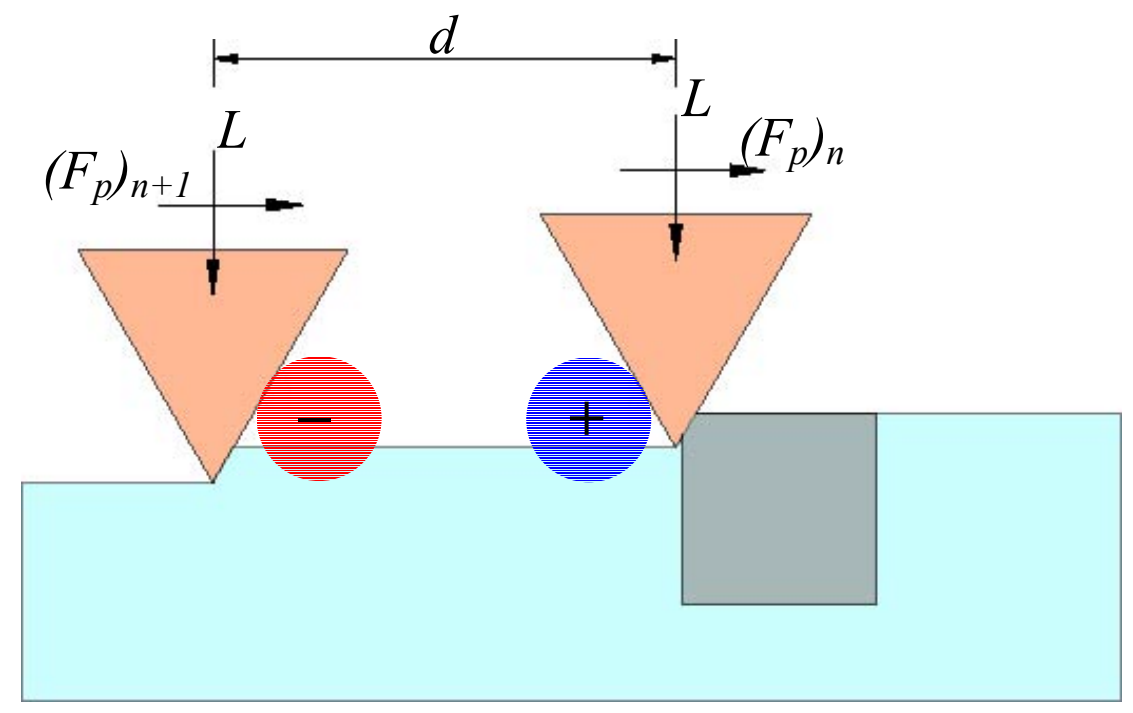

Fig. 3: Abrasive particles plow matrix and reinforcement alternatively and stress fields are formed around them; compressive stresses are formed in front and tensile stresses behind the abrasive particle.

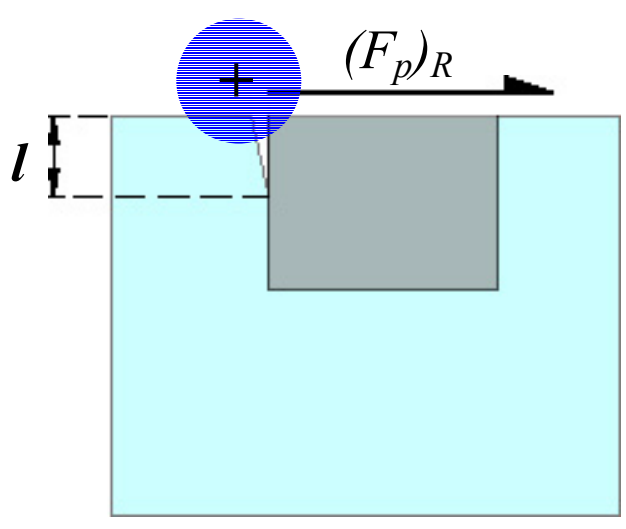

Fig. 4: Delamination of the matrix/reinforcement interface leading to interfacial crack propagation under the tensile loading generated by sliding motion of the abrasive particle. 


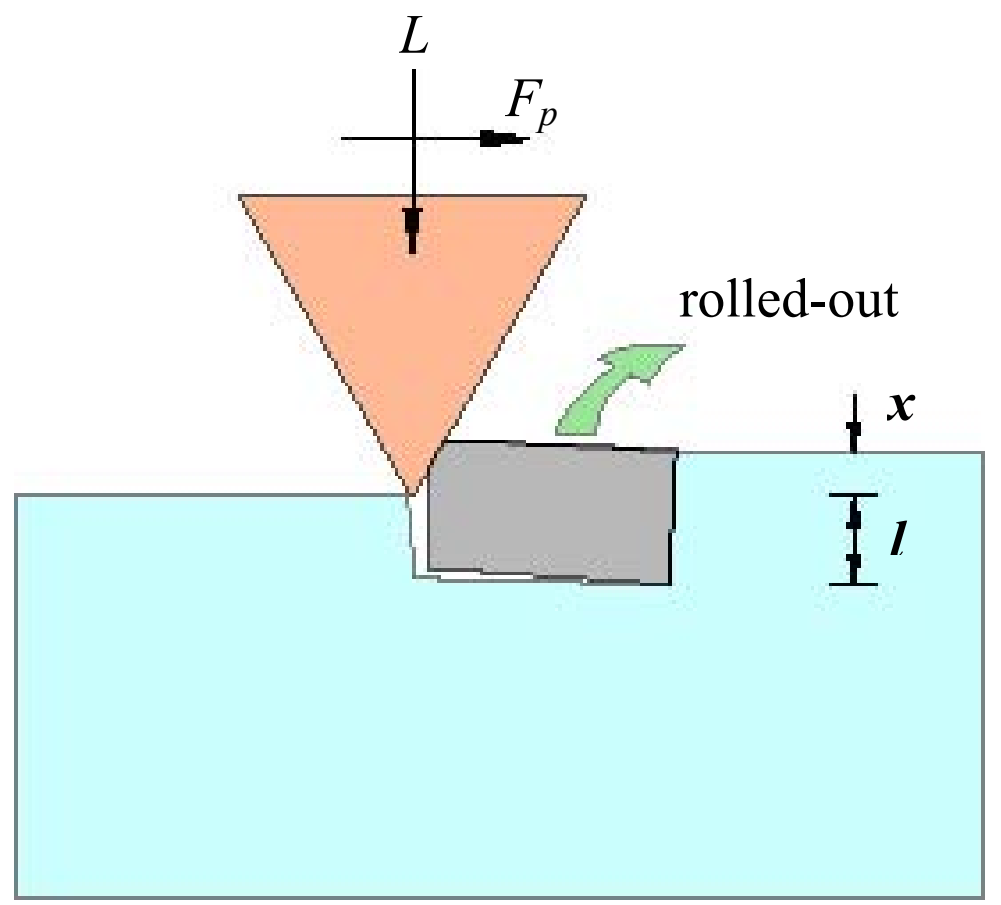

(a)

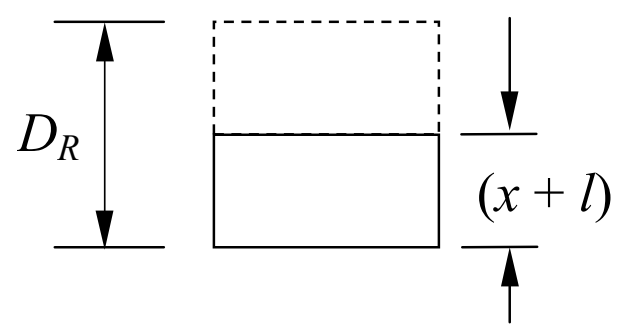

(b)

Fig. 5: A portion of the reinforcement is removed due to interface failure. 



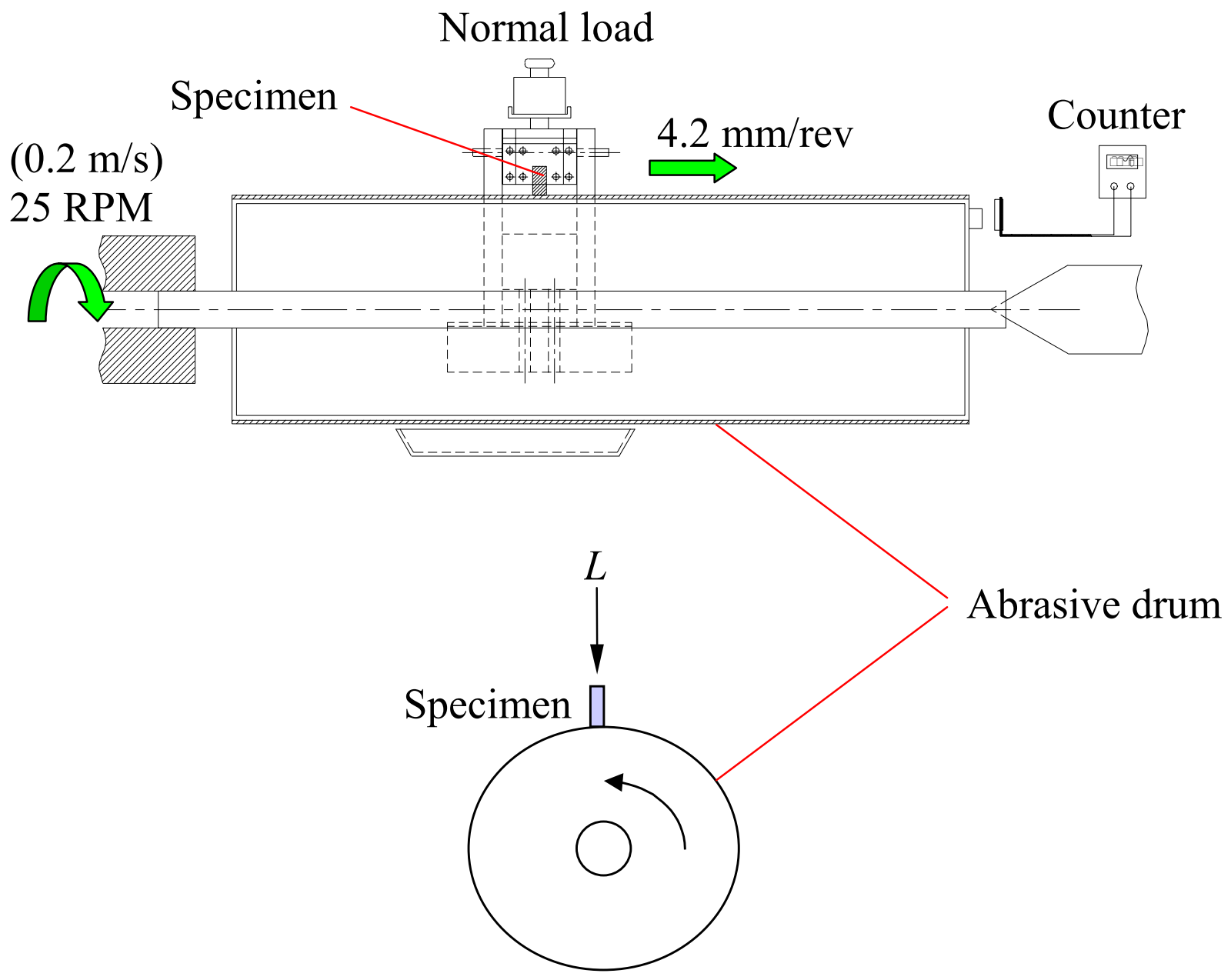

Fig. 6: Pin-on drum abrasive wear test set-up. The drum support is $150 \mathrm{~mm}$ in diameter and rotates at $25 \mathrm{rpm}$. The longitudinal traverse is $4.2 \mathrm{~mm} / \mathrm{rev}$ of the drum. A counter records the number of revolutions. The composite specimen is loaded onto the drum with a specified weight. 

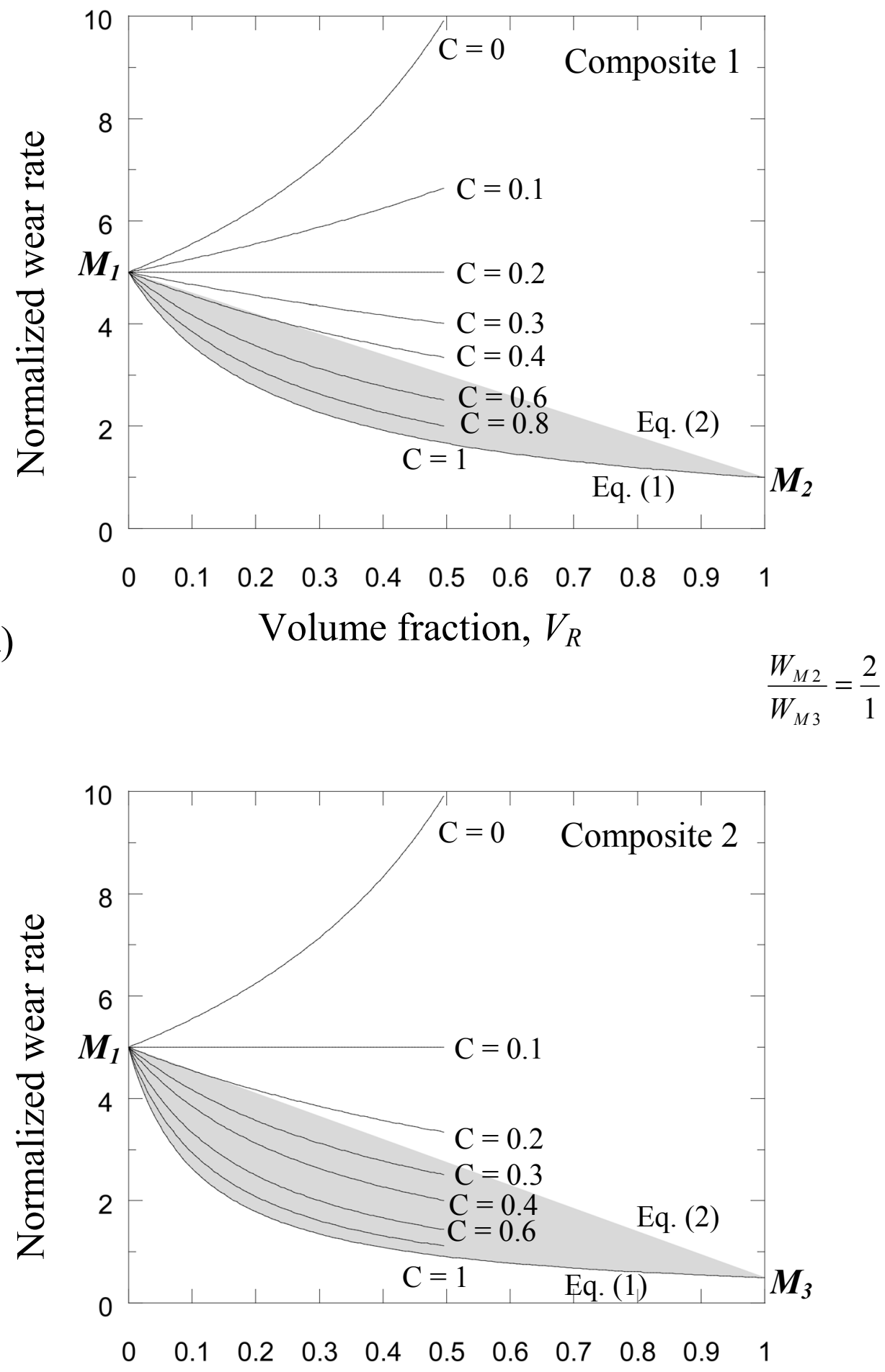

(b)

\section{Volume fraction, $V_{R}$}

Fig. 7: Prediction of abrasive wear rates, normalized to the wear rate of the reinforcement, for two ideal composites with different contribution coefficients and volume fractions. The two composites, termed composite 1 and 2, differ in their relative wear resistance; the abrasive wear resistance of the reinforcement in composite 2 is twice as large as that of the reinforcement in composite 1. Plots are for various values of the contribution coefficient, 
$C$. The shaded region represents the predictions from the rule of mixtures models, specifically Eqs. (1) and (2). 

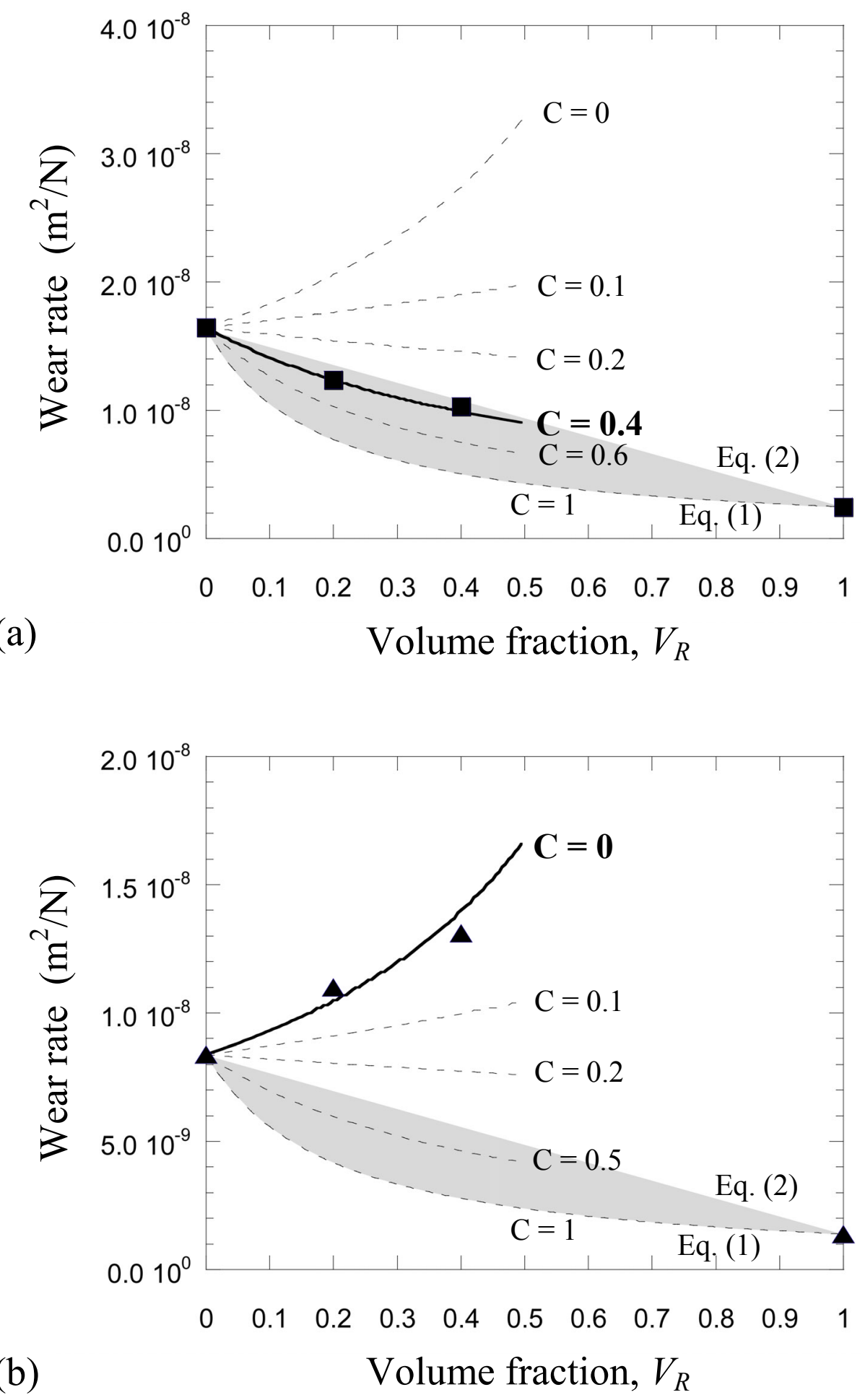

Fig. 8: Abrasive wear rates of particulate reinforced model composites with different interfacial properties between matrix and reinforcement, showing predictions for a) a strong interface $\left(l<<D_{R}\right)$, and b) a very weak interface $\left(l \sim D_{R}\right)$. The curve through the experimental results for Al-reinforced epoxy matrix composites are shown as a solid line; predictions are dashed lines. The shaded region represents the predictions from the rule of mixtures models, specifically Eqs. (1) and (2). 


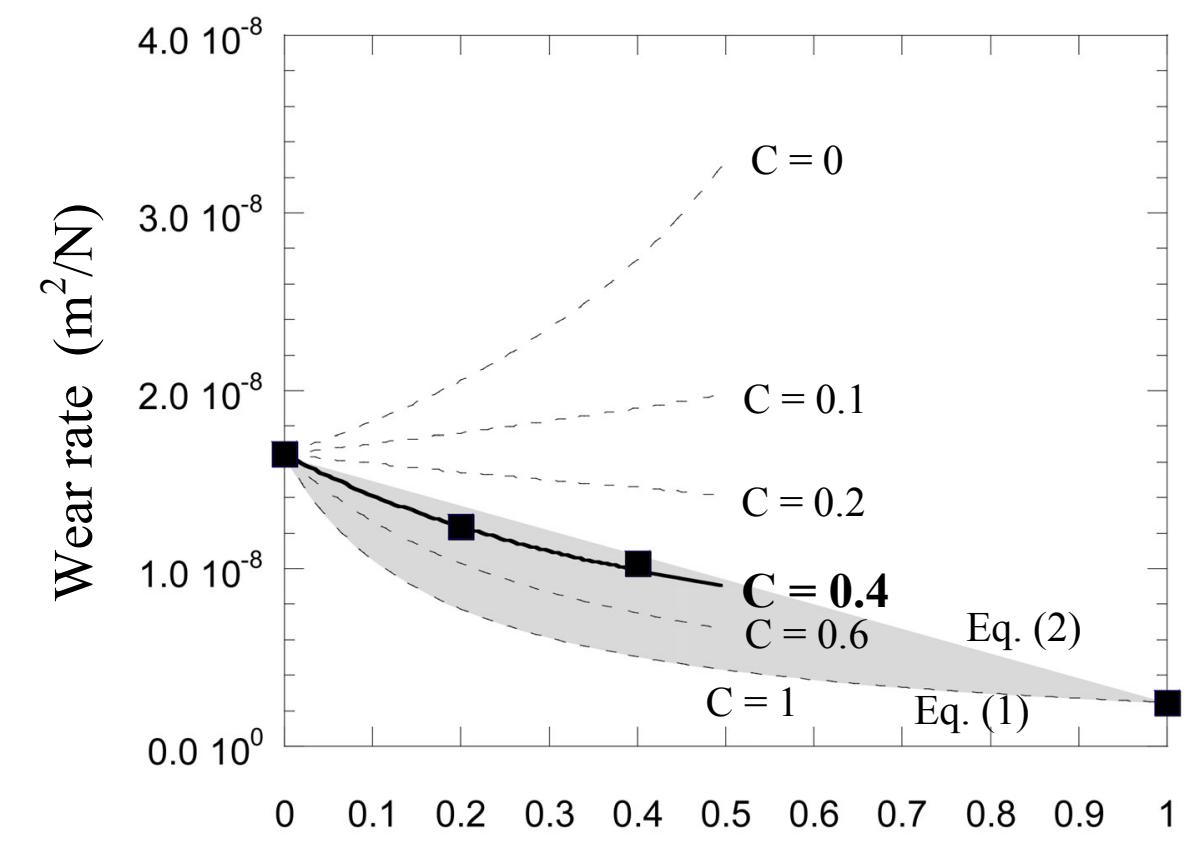

(a)

\section{Volume fraction, $V_{R}$}

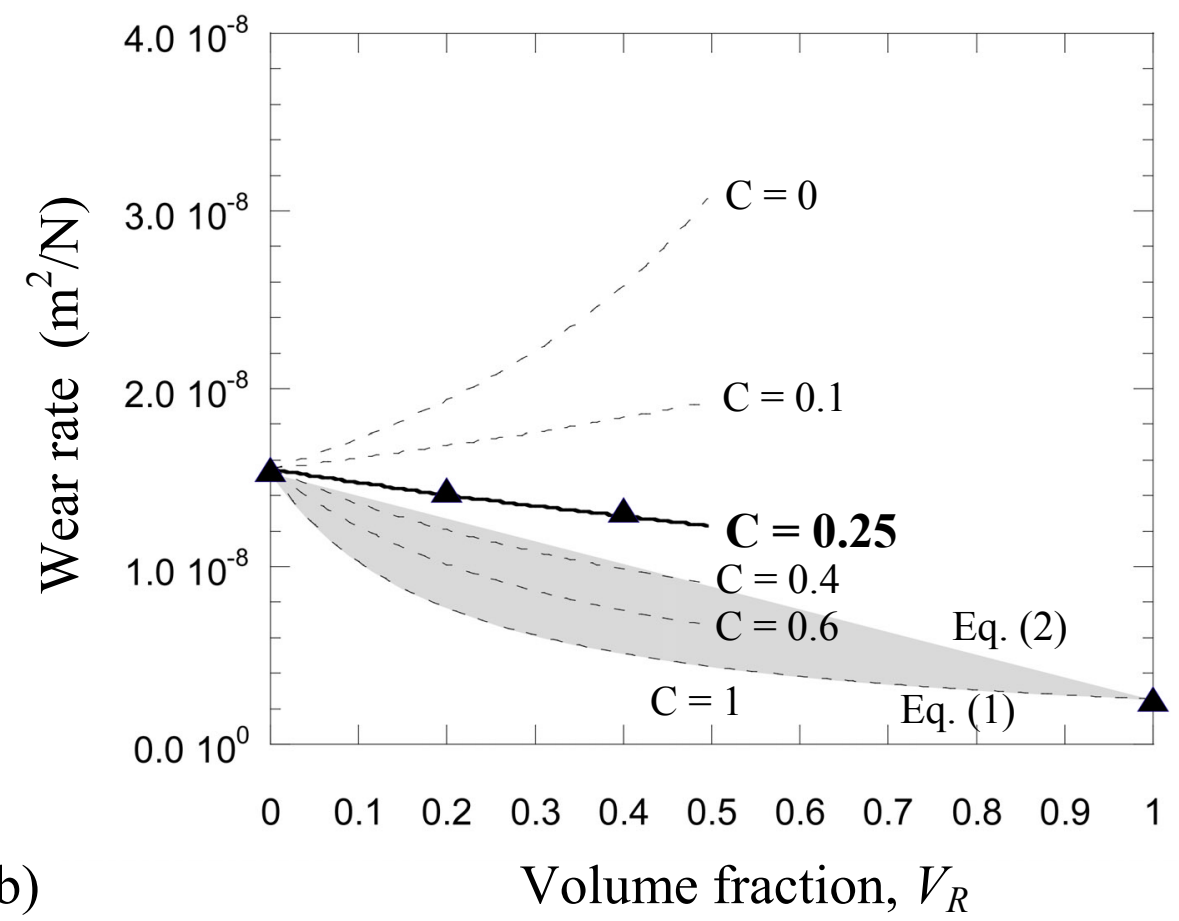

Fig. 9: Abrasive wear rates with different relative size of the reinforcement relative to the abrasive medium, showing experimental results for Al-reinforced epoxy composites (solid lines with data points) and model predictions (dashed lines) for composites with a) large $\left(D_{R}>x\right)$ reinforcements, and b) small $\left(D_{R} \sim x\right)$ reinforcements. The 
shaded region represents the predictions from the rule of mixtures models, specifically Eqs. (1) and (2). 